\title{
Left behind: The potential problem of residual cancer
}

\section{Bobby Shayegan, MD, FRCSC}

Associate Professor, Head, Surgical Oncology SJHH, Deputy Chief of Surgery SJHH, Chair, Surgical Robotic Programme SJHH, McMaster Institute of Urology \& Juravinski Cancer Centre, Department of Surgery, McMaster University, Hamilton, ON

See related article on page 35 .

Cite as: Can Urol Assoc J 2013;7:39. http://dx.doi.org/10.5489/cuaj.239

S ivalingam and Drachenberg report on their retrospective experience in incidentally discovered urothelial and adenocarcinoma of the prostate in cystoprostatectomy specimens. ${ }^{1}$ In keeping with previous reports, the incidence of clinically significant prostate and urothelial cancer was $19 \%$ and $16 \%$, respectively. While no long-term survival data are presented, the authors conclude that the incidence of cancer in the prostate may preclude prostatesparing cystectomy in men with urothelial cancer of the bladder.

It is generally accepted that treatment paradigms for bladder cancer should maximize efficacy while minimizing functional impact or toxicity. Within the context of radical bladder surgery, such an approach would equate to maintaining local and distant cancer control while preserving continence and potency. However, despite advances in the understanding of pelvic surgical anatomy and techniques in orthotopic bladder replacement, maintaining sexual function after nerve-sparing cystoprostatectomy remains a significant challenge. To this end, many investigators have proposed the concept of "prostate-sparing cystectomy" in carefully selected patients at low risk of prostatic adenocarcinoma and urothelial carcinoma. In such a patient population, surgical preservation of the prostate, neurovascular bundles and seminal vesicles along with orthotopic bladder replacement would be expected to increase the probability of maintaining erectile and urinary function. Although, there are favourable reports of long-term cancer control in such a cohort, the potential impact of synchronous or metachronous cancer in the retained prostate cannot be overstated. Local recurrence of urothelial carcinoma is typically diagnosed in a delayed fashion leading to adverse outcomes. Similarly, treatment options for prostate adenocarcinoma are substantially limited by virtue of the prior pelvic surgery. As pointed out by the authors, patients who stand to lose the most are indeed those chosen for prostate-sparing cystectomy as they tend to be the youngest.

To date, survival and functional data on prostate-sparing cystectomy remain biased and difficult to extrapolate. While we must always strive to improve functional outcomes of bladder cancer surgery, oncologic efficacy cannot be compromised. Ultimately, the role of prostate-sparing cystectomy can only be elucidated in prospective randomized trials that eliminate inherent biases. Unfortunately, such a task will be difficult to undertake and unlikely to take place.

Competing interests: None declared.

\section{Reference}

1. Sivalingam $S$, Drachenberg D. The incidence of prostate cancer and urothelial cancer in the prostate in cystoprostatectomy specimens in a tertiary care Canadian centre. Can Urol Assoc J 2013;7:35-8. http:// dx.doi.org/10.5489/cuai.212

Correspondence: Dr. Bobby Shayegan, St. Josephs Healthcare, Institute of Urology, 50 Charlton Ave E, Room G339A, Hamilton, ON L8N 4A6; Email: shayeb@mcmaster.ca 